\title{
Management of Gastro-intestinal Neuroendocrine Tumors with Liver Metastases: A Comprehensive Review
}

\author{
Aich Ranen Kanti*, Ray Amitabh, Das Chaitiparna, Bhattacharya Jibak and Gangopadhyay Subir
}

Department of Radiotherapy, N.R.S. Medical College, Kolkata West Bengal, India

\begin{abstract}
Neuroendocrine tumors have been studied for over a century and several advances have been made in understanding of the pathophysiology, diagnostic methods, management, and underlying genetics. Advances in pathology and identification of serum markers have helped to identify subsets of tumors. Diagnostic modalities like somatostatin receptor scintigraphy (SRS) with CT fusion imaging, Endoscopic ultrasonography (EUS), PET scan and MRI have vastly improved the diagnosis of these tumors. The management of these tumors requires a multidisciplinary approach including endocrinology, interventional radiology, medical surgery, and medical \& radiation oncology. The aggressive use of curative and cytoreductive surgery, orthotopic liver transplantation as well as interventional radiological techniques including embolization, chemo-embolization, and radiofrequency ablation in conjunction with judicial use of somatostatin analogues for symptom control has become the frontline of treatment. For the vast majority of patients with unresectable metastatic disease, older chemotherapeutic agents have shown disappointing results, yet new regimens and new classes of drugs hold great promise.

Clinicians need to be aware with the disease pattern, natural history and disease progression, which are characteristic of these tumors. A multidisciplinary approach is the evidence based logical approach to treat these diseases.
\end{abstract}

Keywords: Neuroendocrine tumors, Hepatic metastasis, Medical management, Metastatic disease, NETs, Newer approaches.

\section{INTRODUCTION}

Neuroendocrine tumors (NET) are a genetically diverse group of malignancies arising from the secretory cells of the neuroendocrine cell system and may be symptomatic (functional) or asymptomatic (non-functional). Until recently they were believed to be derived from migrated neural crest cells but, in fact, they actually arise from local multipotent gastrointestinal stem cells, rather than by migration of the neural crest cells, and hence are endodermal in origin [1]. But both types may frequently synthesize more than one peptides producing characteristic hormonal syndrome.

They were first described in 1888 by Lubarsch [2]. NETs account for less than $1 \%$ of all malignancies; however, Surveillance, Epidemiology, and End Results (SEER) program data suggested that the incidence of malignant NETs is gradually increasing, although this may partly be due to increased physician's awareness as well as improved diagnostic techniques. In 2004 there were 5.25 new cases of NETs per 100000 people, compared with 1.09 per 100000 in 1973 (age-adjusted incidence) [3]. A statistically significant increase in incidence across all disease stages at diagnosis $(P<$ 0.001) has been demonstrated by a time-trend analysis [4]. Neuroendocrine tumors were previously thought to be relatively benign, but are now considered one of increasing malignancy. These tumors are typically indolent and may not become clinically apparent until metastatic spread has occurred or symptoms of carcinoid syndrome appear.

*Address correspondence to this author at the Department of Radiotherapy, N R S Medical College \& Hospital, Kolkata-700014, West Bengal, India; Tel: 9830341692; Fax: 03325537833; E-mail: ranenaich@rediffmail.com
Diagnosis is therefore often made late as the early symptoms are easy to overlook [5].

Carcinoid tumors are a subtype of neuroendocrine tumors. Like other NETs, they also arise from amine precursor uptake and decarboxylation (APUD) cells and express cell membrane neuroamine uptake mechanisms and specific receptors. Carcinoid tumors have historically been classified according to their presumed derivation from different embryonic divisions of the gut. Foregut carcinoid tumors most commonly originate in the thymus, lungs, bronchi, stomach, duodenum, common bile duct and pancreas; midgut tumors in the distal small intestine, appendix and ascending colon; and hindgut tumors in the transverse colon, descending colon and rectum. Carcinoid syndrome is most frequently associated with midgut carcinoid tumors. The characteristics of carcinoid tumors arising from the various subsites are compared in Table 1 [6].

Over $90 \%$ of all carcinoid tumors originate in the gastrointestinal (GI) tract which is considered to be the largest neuroendocrine organ in the body. Unlike other endocrine organs, the endocrine cells of the gut are dispersed as single cells from the gastro-oesophageal junction to the rectum. These cells are part of the amine precursor uptake and decarboxylation system and give this tumor its most distinctive feature: its ability to secrete biogenic amines and polypeptide hormones that cause the carcinoid syndrome [7]. The carcinoid syndrome is usually a result of metastases to the liver with the subsequent release of hormones (serotonin, tachykinins, and other vasoactive compounds) directly into the systemic circulation. PNETs are the most common functioning NETs and they cause various well-established syn- 
Table 1. The Characteristics of Carcinoid Tumors Arising from the Various Subsites

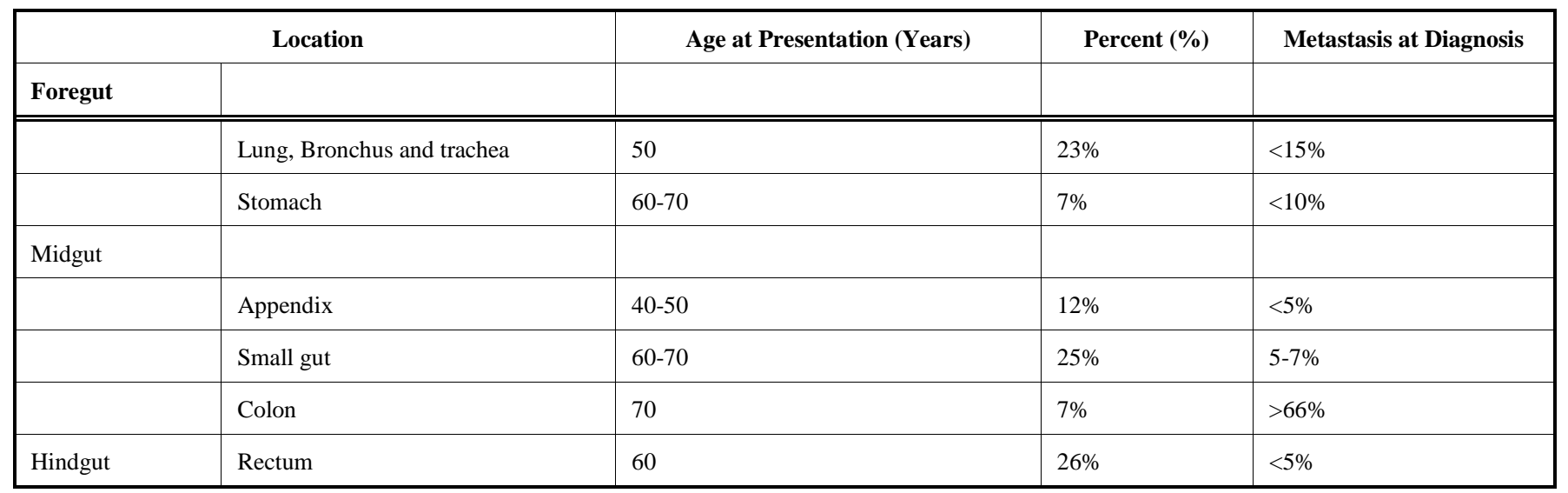

dromes. For example, gastrinomas, more commonly found in the duodenum, cause Zollinger-Ellison syndrome, characterized by peptic ulcers, diarrhea and abdominal pain. Glucagonomas produce excess glucagon, which leads to hyperglycemia and is associated with diabetes mellitus, thrombosis, anemia and atypical skin rash. Insulinomas cause hypoglycemia due to excess insulin, whilst VIPomas produce excess VIP leading to Verner-Morrison syndrome with profuse secretory diarrhea, hypokalemia and metabolic acidosis; whereas somatostatinomas can present with steatorrhea, achlorhydria, diabetes mellitus, and cholelithiasis. Extrapancreatic somatostatinomas can occur in the duodenum, cystic duct, colon, and rectum. These tumors present more often due to the mass effect, with obstruction and pancreatitis, although occasionally diabetes and cholelithiasis have been described.

\section{PATHOLOGICAL FEATURES}

Carcinoid tumors are usually composed of uniform cells with moderate amounts of granular cytoplasm and round nuclei. The embryonic origin of carcinoids is the neuroectoderm argentaffin cells located in the mucosa near the base of the crypts of Lieberkuhn. There are five major histological patterns: (1) insular; (2) trabecular or ribbon; (3) tubular or rosette-like; (4) atypical or poorly differentiated; and (5) mixed. These patterns correlate with the embryologic location: foregut-origin tumors show a trabecular growth pattern, midgut tumors tend to grow with an insular pattern, and hindgut tumors show mixed patterns of growth [7].

Carcinoid tumors can be identified by silver impregnation stains, either argyrophilic or argentophilic. Because carcinoid tumors produce numerous polypeptides and hormones, immunohistochemistry markers are found for serotonin, gastrin, glucagon, somatostatin, insulin, substance $\mathrm{P}$, and pancreatic polypeptide [7].

The distinction between benign and malignant carcinoid tumors is more difficult than with other GI epithelial tumors. Because individual cells making up the tumor usually appear bland, and only rarely show mitoses or hyperchromatism; the diagnosis of malignancy requires gross or microscopic evidence of invasion. All carcinoid should be considered potentially malignant, even though the majority is less than $2 \mathrm{~cm}$ in size and those found incidentally are very likely to be benign. Factors that determine malignant behavior include: (1) presence of invasion; (2) tumor size; and (3) site of origin. Metastases occur in only $50 \%$ of patients with tumors smaller than $1 \mathrm{~cm}$, but 95\% when tumors are larger than $2 \mathrm{~cm}$ [8].

Carcinoid tumors spread locally to regional lymph nodes, liver, other intra-abdominal organs and lung, but only rarely to bone. Only a tiny minority of those that arise in the stomach or rectum spread beyond their site of origin. Appendiceal carcinoids almost never metastasize because even small tumors obstruct the lumen to produce appendicitis early, prompting surgical removal before metastases occur. Small carcinoids normally do not invade or obstruct the bowel lumen but can penetrate the muscle layer, causing marked desmoplasia that may lead to adhesions, bowel kinking and angulation, and obstruction [6].

Massive fibrosis of the mesenteries, omenta, and peritoneum may result from the leakage of serotonin and other vasoactive substances independent of any intrinsic disease. This fibrosis is responsible for the fixation, kinking, and angulations seen on small bowel series and the stellate soft tissue density mass on CT and MRI scans [9-11].

\section{LIVER METASTASIS IN CARCINOID TUMORS}

Liver metastases from carcinoid tumors may be found synchronously with the primary tumor or metachronously at follow-up after resection of the primary tumor, or may occur even in the absence of a detectable primary tumor. The diagnosis of NETs is multimodal, based on clinical findings, radiological and nuclear imaging, hormone levels estimation, and histological confirmation. Clinical symptoms generally appear after the NETs have metastasized.

\section{IMAGING}

Many patients present with metastatic disease with no known primary site. Opinion is divided on whether locating the primary changes prognosis. Investigations for localizing the primary site may include (depending on the type of tumor and symptoms): ultrasound scans of the abdomen, testes, and ovaries; Endoscopic ultrasonography (EUS); somatostatin receptor scintigraphy (SRS), portal venous sampling, and angiography; CT scan of the chest (bronchial 
carcinoid), abdomen, and pelvis; upper GI-endoscopy and colonoscopy; barium studies; and nuclear medicine functional imaging. Still primary tumors may be very difficult to detect. EUS is a major diagnostic investigation in a patient with a suspected pancreatic NET. Its sensitivity may be less with extrapancreatic gastrinomas $(80 \%$ of gastrinomas in MEN1 are found in the duodenum) for which an upper gastrointestinal endoscopy and CT or MRI should be preferred [12]. In one series, primary tumors were localized in 81$96 \%$ of cases using radiological and/or nuclear medicine imaging [13]. Ultrasonography, CT, and MRI, respectively has been found to detect only $9 \%, 31 \%$, and $20 \%$ of primaries [14].

Previously, portal venous sampling was used to facilitate identification of functional primary tumors with a reported sensitivity of $62 \%$ (or $92 \%$ for pancreatic insulinomas using arterial stimulation with calcium), but this has been replaced by SRS and EUS. SRS with its high affinity to the two most prevalent somatostatin receptors found on GI-NETs ( $\mathrm{sst}_{2}$ and sst $_{5}$ ) —allows visual evidence of GI-NET localization. SRS is indicated as the first staging procedure and is one of the most sensitive single-screening methods for extrahepatic disease manifestation [15]. With the exception of insulinomas (50\% of tumors express SSTR2), SRS plays a central role in locating and assessing the primary in gastroenteropancreatic NETs. For gut tumors, a sensitivity of up to $90 \%$ has been noted with SRS. The sensitivity could be further enhanced by the use of single positron emission computed tomography and fusion imaging with CT. Similarly, when the primary tumor has been resected, SSR may be the tool of choice for diagnosis of recurrence. However, in patients whose octreoscan is negative and in whom no diagnosis is reached after upper and lower gastrointestinal endoscopy, a triple phase CT scan of the thorax and abdomen is regarded as the investigation of choice. Positron emission tomography (PET)-CT scan imaging and single photon emission computed tomography-CT hybrid systems have been used in several malignancies with high uptake of ${ }^{18} \mathrm{~F}$-fluorodeoxyglucose but are not useful for NETs except for the aggressive tumors. Recent advances in PET imaging using ${ }^{18} \mathrm{~F}$-levodopa for carcinoid tumors and carbon- $11\left({ }^{11} \mathrm{C}\right)$ tryptophan for recurrences and visually occult tumors provide complimentary diagnostic information to other imaging modalities $[16,17]$. Advances in the use of gallium-68 labeled agents ([ ${ }^{68} \mathrm{Ga}-$ DOTA]-D-Phe(1)-Tyr(3)-octreotide) to identify metastatic NETs, while allowing for the performance of scans one hour after injection, has been particularly exciting and hold promise for the possible detection of primary tumors. PET scanning with gallium 68-DOTATOC is likely to replace SRS in future [18].

Somatostatin receptor scintigraphy combined with CT/MRI increased detection rates for primary tumors to $68 \%$; [19] while primary tumors in the pancreas as small as 5 $\mathrm{mm}$ are reliably detected by EUS. [14] Liver metastases may be detected using US, CT, and MRI with sensitivities of $46 \%, 42 \%$, and $43 \%$, respectively [14]. In the past, selective angiography was used to detect hepatic metastases and had a reported sensitivity of $86 \%$ but this is no longer used, since the combination of SRS and CT/MRI detects $96 \%$ of liver metastases [19].
On CT, carcinoid metastases to the liver have a characteristic appearance due to their hypervascularity. They are hypodense on non-contrast scans and demonstrate robust enhancement on early hepatic arterial-phase imaging following the administration of intravenous contrast material. These lesions may become isodense with normal hepatic parenchyma in scans obtained during the portal venous or equilibrium phase. Accordingly, if metastatic carcinoid tumor is suspected, hepatic arterial phase imaging should be obtained [14].

On MRI, most hepatic metastases from carcinoid tumors are hypointense on T1-weighted, and moderately or strongly hyperintense on T2-weighted unenhanced scans. Following the intravenous administration of gadolinium, these tumors show intense enhancement during hepatic arterial-phase imaging. These lesions tend to become isointense to normal liver during scans obtained during the portal venous and equilibrium phases. Indeed, $10 \%$ of hepatic metastases were seen only on the arterial phase. [11] The use of dynamic contrast-enhanced MRI using contrast agents like ultrasmall superparamagnetic iron oxide particles is sensitive to the vascular phase of contrast medium delivery and can demonstrate vascular permeability and assess lymph nodes. New agents including gadolinium-EOB-DTPA (gadoxetate) and SHU-555A are being used for liver imaging. Gadoxetate is selectively taken up by hepatocytes, which increases the signal intensity on T1-weighted images, leading to better lesion-to-liver contrast, whereas SHU-555A in the reticuloendothelial system causes a decrease in the signal intensity of the liver parenchyma on both T2- and T1weighted images, although it is unchanged in metastatic tumors, improving the lesion-to-liver contrast. Molecular MRI with antibodies or gadolinium-labeled peptides can detect receptors on tumors and identify tumoral antigens such as ErbB2 and can assess the efficacy of antitumor agents.

Larger carcinoid metastases may show heterogeneous enhancement, central necrosis and occasionally, peripheral enhancement with progressive fill-in, but without globular enhancement [10].

\section{FINE-NEEDLE ASPIRATION CYTOLOGY/CORE BIOPSY}

Although preoperative diagnosis of suspected neuroendocrine metastases may be attempted by fine-needle aspiration cytology (FNAC), in a recent study the correct diagnosis was obtained in only a third of cases by this method, due to similarities between the cytological features of NET and adenocarcinomas [20]. Since the decision to operate or not to operate depends on the pattern of disease and its suitability for resection, FNAC or core biopsy is only indicated in patients in whom "curative resection" is not an option, but histological diagnosis is required. Diagnostic biopsy may be indicated to aid selection of patients for liver transplantation.

\section{METABOLIC TUMOR MARKERS}

Carcinoid tumors, like other APUD tumors, contain proteins and release hormones which are metabolized and excreted. A variety of biochemical tests are available for symptomatic patients, some are specific and some non-specific, which can assist with the initial diagnosis and assessment of 
required treatment, in monitoring disease progression and treatment response and may offer prognostic information. Neurosecretory granules within the carcinoid tumors release serotonin that is metabolized into 5-HIAA and excreted in the urine. In addition, Chromogranin A (CgA), a 49-kDa protein, has been identified as being contained in the neurosecretory vesicles of the tumor cells and is detectable in the plasma of patients with carcinoid tumors and other peptide hormone-producing tumors [5]. Studies have suggested CgA as the primary biomarker for the diagnosis of NETs as levels correlate with tumor burden [21], and provide a more sensitive assessment compared with other biomarkers [22]. In addition, early CgA response (normalization or $\geq 30 \%$ decrease by week 4) may correlate with improved progression-free survival [23]. The technique used for determination of CgA might affect its sensitivity because GEP-NETs might have a higher cleavage of CgA; therefore, enzyme-linked immunoadsorbent assay might be more sensitive than immunoradiometric assay. Levels of circulating CgA are increased in $60 \%$ to $80 \%$ of patients with GEP-NETs but are nonspecific, due to elevation in renal failure, proton pump inhibitor use, and chronic atrophic gastritis.

CgA and synatophysin are recognized as universal stains applicable for all NETs, but the applicability of immunohistochemistry for well-differentiated tumors and the use of CgB, NCAM, CD57, neuron-specific enolase, and keratins are not routinely recommended. The role of CDX2 and TTF1 in determining the site of origin and the role of Ki-67 in determining proliferative potential are recognized, but uniform guidelines for their estimation are yet to be developed.

\section{MANAGEMENT OF NETS WITH LIVER METASTA- SIS}

Liver metastasis can occur in $50 \%$ to $75 \%$ of small bowel NETs, $5 \%$ to $70 \%$ of foregut NETs, $14 \%$ of hindgut NETs, and $30 \%$ to $85 \%$ of PNETs [4]. The aim of treatment should be curative where possible but is palliative in the majority of cases. These patients often maintain a good quality of life for a long period despite having metastases. Treatment of metastatic carcinoid to the liver has two distinct targets: (1) the tumor with its metastases, and (2) the carcinoid syndrome.

\section{SURGICAL MANAGEMENT}

Overall survival in patients with carcinoid tumors depends on control of tumor growth and palliation of symptoms related to vasoactive intestinal amines. Surgery plays the key role in the management of NETs even in the presence of liver metastasis. Those patients presenting with suspected appendicitis, intestinal obstruction, or other gastrointestinal emergencies are likely to require resections sufficient to correct the immediate problem. Once definitive histopathology is obtained, a further more radical resection may have to be considered. The approach to curative resection is similar to that for adenocarcinoma. In the case of small bowel tumors, a limited emergency small bowel resection for an obstructing carcinoid tumor can be followed at a later date by elective surgery to remove further small bowel. This is particularly appropriate if by then a second tumor has been identified, or to undertake mesenteric lymphadenectomy. A substantial minority of patients with midgut carcinoid have multiple tumors, so a search should be made following removal of an obstructing lesion prior to any further surgery. Terminal ileal tumors mandate ileo-cectomy and radical regional lymph-node resection. Carcinoids in the remainder of the ileum and the jejunum are treated with wide segmental resection. A Whipple's procedure is often performed for duodenal carcinoids. Tumors that cause bowel symptoms and asymptomatic tumors larger than $1 \mathrm{~cm}$ should be managed as if they were frankly malignant and capable of metastasis. Asymptomatic carcinoids less than $1 \mathrm{~cm}$ in size may only need local excision [24].

In patients with gastric carcinoid the approach depends on the type of tumor of which there are three types. Type 1 gastric carcinoids originate from enterochromaffin-like cells, associated with hypergastrinaemia and chronic atrophic gastritis, and can synthesize and store histamine. The frequency of metastasis is low, and in many cases surveillance only is appropriate, although limited surgery with endoscopic polypectomy and/or antrectomy may be preferable. Type 2 gastric carcinoids occur in patients with hypergastrinaemia due to Zollinger-Ellison syndrome in combination with MEN type1. Type 3 gastric carcinoids are sporadic, not associated with hypergastrinaemia and have a more malignant course. These tumors have often metastasized by the time of diagnosis. Small tumors less than $1 \mathrm{~cm}$ with no extension into muscle on EUS or CT could be resected endoscopically but most lesions will need resection and clearance of regional lymph nodes.

Pancreatic and periampullary NETs require particular consideration. For insulinoma, if the lesion is clearly localized before surgery, and is near or at the surface of the pancreas and easily defined at surgery, enucleation may be sufficient, provided histopathology demonstrates complete excision and benign features. However, this may not be possible and Whipple's pancreatoduodenectomy, left pancreatectomy, or even total pancreatectomy may be justified in selected cases. These operations are also applied to selected cases with localized disease arising from other functioning, as well as non-functioning, NETs of the pancreas.

Colo-rectal carcinoids should be treated with adequate surgical resection and pelvic lymphadenectomy. Metastatic lymph node removal may contribute to long term survival.

Patients with the carcinoid syndrome already have widespread metastatic disease. The survival varies from $40 \%$ to $83 \%$ at 5 years, with a relatively low operative mortality (0\%-5.3\%) [20]. However, in studies with longer follow up, it is clear that most patients develop disease recurrence, with a recurrence rate of almost $94 \%$ at 10 years [25]. Nevertheless, the reported survival rates after surgical resection of metastases remain higher than those reported for medical therapy alone, but the data are not conclusive.

Liver metastasis needs special attention as most of the patients with NETs have liver involvement at the time of presentation. Most authorities recommend even debulking of unresectable hepatic and intestinal metastatic carcinoid lesions both to help alleviate some of the symptoms of carcinoid tumor and, according to some reports, to actually improve survival. Because most patients with carcinoid syndrome have diffuse metastases, hepatic artery embolization 
and hepatic dearterialization procedures have proved very helpful [26].

Unlike hepatic metastases from most malignancies that lead to hepatic dysfunction and failure, this is rarely a complication of hepatic carcinoids despite massive hepatic replacement by tumor. The liver metastases are usually large, bulky and encapsulated with displacement rather than encasement and invasion of adjacent vasculature and bile ducts [27]. This makes surgical resections more feasible and probably less extensive, because parenchyma sparing wedge resections or segmentectomies may be sufficient. Surgical removal of hepatic metastases is the only curative treatment and should always be considered. In the presence of liver metastases, 'curative' liver resection is possible in approximately $10 \%$ of cases if the lesion(s) is (are) confined to one lobe. In the remaining patients, palliative resection may still be an option in order to minimize endocrine or local symptoms and to improve the effect of adjuvant medical therapy and is possible in another $10-15 \%$ of the patients. The five year survival after resection of the primary and/or liver secondary is up to $87 \%$ and postoperative mortality is $6 \%$ [27]. Several series have shown low morbidity and excellent medium term survival after liver resection with worse outcomes in other patients not resected; [28] but this may partly reflect stage of disease. A minority of patients with no obvious primary may have primary hepatic neuroendocrine malignancy and for such patients, surgery is the treatment of choice, with a recurrence rate of $18 \%$ and five year survival of $74 \%$ reported in one series [29]. Many patients will need somatostatin analogues which predispose patients to gallstones; hence the gall bladder is also usually removed at the time of liver surgery. The Mayo Clinic recommends palliative resection if more than $90 \%$ of hepatic metastases is resectable and the extra hepatic tumor bulk is limited [26]. Hepatic resection can be performed in patients with bilobar disease but should be avoided in cases with extensive hepatic involvement (>75\%) or with widespread extra hepatic disease, as the post-operative morbidity and mortality are too high $[23,26]$. Severe carcinoid heart disease with elevated central and hepatic venous pressure may cause serious intraoperative bleeding and is a relative contraindication to hepatic surgery. These patients may benefit from cardiac valve replacement prior to hepatic resection [30].

Orthotopic liver transplantation (OLT) has been applied to relieve symptoms, either due to tumor mass or hormone release from unresectable neuroendocrine liver metastases, which is currently viewed as the only metastatic indication for transplantation [31]. Five-year actuarial and disease-free survivals of $36 \%$ to $73 \%$ and $24 \%$ to $52 \%$, respectively, have been observed after OLT for metastatic neuroendocrine tumors [32], with symptomatic relief occurring in $90 \%$ to $100 \%$ of patients [27]. Actuarial 5-year and disease-free survivals of $70 \%$ and $53 \%$, respectively, have been achieved using the Milan criteria which excludes patients with noncarcinoid tumors or extrahepatic disease [33].

Milan criteria for suitability of liver transplantation in NETs with liver metastases include
1. Confirmed carcinoid histology
2. Primary tumor drained by portal system

3. $>50 \%$ hepatic replacement by tumor metastases

4. Good response or stable disease during the pretransplantation period

\section{Absence of extrahepatic disease}

Although these criteria must be validated before universal application, improved patient selection and use of OLT earlier in the disease course would seem to be a logical approach. Till date approximately 150 patients with metastatic liver NETs have received such a transplant. Due to organ shortage, this should not be used in general but might be considered in exceptional circumstances. Further research is needed to try to assess pre-transplantation prognostic factors.

\section{HEPATIC ARTERY EMBOLIZATION}

This procedure is indicated for patients with nonresectable multiple and hormone secreting tumors. Arterial embolization induces ischemia of the tumor cells causing liquefaction, thereby reducing their hormone output. Ischemia of tumor cells also increases their sensitivity to chemotherapeutic substances and this underlies the principle of chemo-embolization. There are two types of embolization: particle and chemo-embolization. Particles used include polyvinyl alcohol (250- to 355- $\mu \mathrm{m}$ polyvinyl alcohol) and gel foam powder (500- to 700- $\mu \mathrm{m}$ microspheres). For chemoembolization, an emulsion of a cytotoxic drug such as doxorubicin $\left(50 \mathrm{mg} / \mathrm{m}^{2}\right)$ or streptozocin $\left(1.5 \mathrm{~g} / \mathrm{m}^{2}\right)$ is used until complete stasis of flow. Individual embolizations are spaced 4 weeks apart and the majority of patients complete their embolizations in 2 or 3 stages [34, 35].

\section{HEPATIC ARTERY RADIO-EMBOLIZATION}

The goal of radio-embolization is to cause tumor necrosis through radiation exposure. Cell death by radiation requires normal oxygen tension. Therefore, stasis of flow is avoided during radioembolization. Infusion of radioembolic agent is typically performed to both lobes rather than staged using a unilobar approach.

In a retrospective multi-institution study, radioembolization using ${ }^{90} \mathrm{Y}$ demonstrated complete imaging response in $2.7 \%$ of patients, a partial response in $60.5 \%$ of patients, stable disease in $22.7 \%$ of patients, progression of disease in $4.9 \%$ of patients, and a median survival of 70 months [36].

\section{RADIOFREQUENCY ABLATION}

Most patients with neuroendocrine metastases have a large number of small metastases that are hormonally active. Radiofrequency ablation is a relatively new treatment modality that has been used with some success in stabilizing or reducing tumor size and to reduce hormone secretion. It may be indicated in patients with inoperable bilobar metastases in whom hepatic artery embolization has failed [37]. It can be performed percutaneously or laparoscopically and the formal approach is preferred, as it is least invasive, cheaper, and has the additional benefit of CT or MRI guidance. The laparoscopic approach has the benefit of intraoperative ultrasound scanning, which is ideal for the detection of tiny tumors but does require considerable skill [38]. Neuroendocrine metas- 
tases are small, numerous, and very slow growing. Therefore, it is possible to treat patients with indolent disease with as many as 20 small $(<3 \mathrm{~cm})$ tumors at multiple treatment sessions over a period of years. Destroying the largest lesion may not necessarily switch off hormone production. To achieve a reduction in hormone secretion it is necessary to ablate at least $90 \%$ of the visible tumor, [39, 40] and radiofrequency ablation has also been used with success in these tumors [41]. Radiofrequency thermal ablation is a minimally invasive means of achieving destruction of hepatic metastases and has been used to treat NETs with liver metastases. Application of radiofrequency electrical energy directly into a metastasis results in heat-induced denaturation of cellular proteins with consequent cell death. It has provided good palliation of tumors up to $3 \mathrm{~cm}$ diameter with less morbidity than has been reported with chemo-embolization, but its effect on patient survival has yet to be confirmed [42].

\section{CHEMOTHERAPY}

Systemic chemotherapy is often inadequate for treatment of NETs, because these tumors have a well-differentiated histology and low proliferation index. Chemotherapeutic agents such as streptozocin and chlorozotocin have been used to treat metastatic neuroendocrine tumors for some time. However, response rates are variable (36\% to 63\%) and usually of short duration and frequently accompanied by systemic toxicity particularly nephrotoxicity [43]. Fluorouracil (5-FU) and doxorubicin are less toxic than streptozotocin, but the observed objective response rate is quite low, i.e. $\leq 25 \%$ [44]. 5-FU, dacarbazine and anthracyclines in different combinations have shown the high response rates and no cross-resistance, because their mechanisms of action do not overlap [45]. Response to chemotherapy in patients with strongly positive carcinoid tumors was of the order of only $10 \%$ whereas patients with SSRS negative tumors had a response rate in excess of $70 \%$ [46]. The highest response rates with chemotherapy are seen in the poorly differentiated and anaplastic NETs: response rates of $70 \%$ or more have been seen with cisplatin and etoposide based combinations [47]. These responses may be relatively short lasting of only 8-10 months. However, the best results have been seen from the Mayo clinic where up to $70 \%$ response rates with progression free survival for several years have been seen by combining chemoembolisation of the hepatic artery with chemotherapy [48]. Temozolomide alone or in combination with capecitabine or bevacizumab is a new therapeutic concept for PNETs but needs further evaluation [49]. Response rates for pancreatic islet cell tumors vary between $40 \%$ and $70 \%$ and usually involve combinations of streptozotocin (or lomustine), dacarbazine, 5-fluorouracil, and adriamycin. Use of same chemotherapeutic agents for midgut carcinoids has a much lower response rate, with $15-30 \%$ of patients deriving benefit, which may only last 6-8 months. The management of pulmonary carcinoids is more likely to involve a platinum and etoposide combination. Polychemotherapy including 5FU, dacarbazine and epiadriamycin is safe and efficacious for the treatment of carcinoids and other less differentiated and more aggressive NETs e.g. Merkel's cell carcinomas, MTC and pancreatic islet-cell tumors. Similarly, chemotherapy with CCNU and 5-FU, either alone or in combination with other therapeutic modalities, produces considerable symptomatic and hormonal improvement and moderate tumor regression/stabilization particularly in patients with metastatic gastroenteropancreatic neuroendocrine tumors with minimal adverse effects. However, long-term survival is still relatively poor.

\section{TARGETED THERAPY}

Targeted radionuclide therapy is a useful palliative option for symptomatic patients with inoperable or metastatic tumor. The principle of treatment is only to give radionuclide therapy when there is abnormally increased uptake of the corresponding imaging agent. The gamma emitting imaging radionuclide is replaced by a beta imaging therapy radionuclide: ${ }^{131} \mathrm{I}-\mathrm{MIBG}$ for ${ }^{123} \mathrm{I}-\mathrm{MIBG},{ }^{90} \mathrm{Y}$-octreotide for ${ }^{111} \mathrm{In}$ octreotate, and ${ }^{90} \mathrm{Y}$-lanreotide for ${ }^{111} \mathrm{In}$-lanreotide. Treatment indications include evidence of avid uptake of ${ }^{123}$ I-MIBG or ${ }^{111}$ In octreotide at all known tumor sites on diagnostic imaging. Contraindications include pregnancy and breast feeding, myelosuppression, and renal failure (glomerular filtration rate $<40 \mathrm{ml} / \mathrm{min}$ ) [50].

Experience using ${ }^{90} \mathrm{Y}$-DOTATOC is growing although it is not widely available at present. Usual cumulative activities range from 12 to $18 \mathrm{GBq}$ administered in 3-6 GBq fractions at 6-8 week intervals. Most patients report subjective benefit within two treatment cycles, often associated with reduction in biochemical tumor markers. The majority of patients achieve tumor stabilization although significant tumor regression is unusual. Experience with ${ }^{90} \mathrm{Y}$-lanreotide therapy is limited but it is clear that the range of tumors taking up ${ }^{111}$ In-lanreotide differs from those taking up ${ }^{111}$ In-octreotide, and therefore ${ }^{111}$ In-lanreotide imaging is required to select patients for ${ }^{90} \mathrm{Y}$-lanreotide therapy.

In gastroenteropancreatic neuroendocrine tumors (GEPNETs) with metastatic disease, cytoreductive therapeutic options are limited. A relatively new therapy is peptide receptor radionuclide therapy (PRRT) with radiolabeled somatostatin analogs [51, 52].

Patients were treated with radiolabeled somatostatin ana$\log \left[{ }^{177} \mathrm{Lu}-\mathrm{DOTA}{ }^{0}, \mathrm{Tyr}^{3}\right]$ octreotate up to a cumulative dose of 750 to $800 \mathrm{mCi}(27.8-29.6 \mathrm{GBq})$, usually in four treatment cycles, with treatment intervals of 6 to 10 weeks corresponding with a radiation dose to the bone marrow of $2 \mathrm{~Gy}$ ), unless dosimetric calculations indicated that the radiation dose to the kidneys would then exceed $23 \mathrm{~Gy}$; in these cases the cumulative dose was reduced to 500 to $700 \mathrm{mCi}$. Toxicity analysis was done in 504 patients, and efficacy analysis in 310 patients.

Apart from nausea and vomiting, hematological toxicity WHO grade 3 or 4 , occurred in $3.6 \%, 4$ to 8 weeks after of administrations. Serious delayed toxicities were observed in nine patients, mainly myelodysplastic syndrome, liver and renal toxicities. Complete and partial tumor remissions occurred in $2 \%$ and $28 \%$ of 310 GEPNET patients, respectively. Minor tumor response (decrease in size > 25\% and < $50 \%$ ) occurred in $16 \%$. Median time to progression was 40 months. Median OS from start of treatment was 46 months, median OS from diagnosis was 128 months. Compared with historical controls, there was a survival benefit of 40 to 72 months from diagnosis. Significant factors were the presence 
of bone metastases $(P<.001)$, extent of liver involvement $(P$ $=.001)$, and gastrinoma, insulinoma, or VIPoma tumor type $(P<.01)$.

Mammalian target of rapamycin (mTOR) is a central regulator of protein synthesis important in cancer, including cell growth and proliferation, angiogenesis and cell metabolism. Everolimus is an oral, mTOR inhibitor that blocks the mTOR pathway by binding to its intracellular receptor FKBP-12. A synergistic antiproliferative effect may be achieved by combining everolimus with octreotide. Promising antitumor activity and progression free survival (PFS) was observed by applying everolimus and octreotide combination in patients with metastatic low or intermediate grade NETs [23]. Slightly better partial response and PFS rates were observed with a higher dose of everolimus (10 versus 5 mg / day). A phase II trial (RADIANT-1) of everolimus with or without octreotide LAR in patients with advanced PNETs following chemotherapy failure found that in those receiving everolimus monotherapy median PFS was 9.7 months and $59.3 \%$ experienced a stabilization or decrease in tumor size [23]. In contrast, patients receiving everolimus $10 \mathrm{mg} /$ day plus octreotide $\leq 30 \mathrm{mg} / \mathrm{month}$ achieved a median PFS of 16.7 months and $84.2 \%$ had tumor stabilization or shrinkage. RADIANT-2, [53] was a randomized phase III, doubleblind, multicenter trial of combination octreotide LAR plus everolimus versus octreotide LAR plus placebo, in patients with advanced carcinoid tumors. 429 patients with low-grade or intermediate-grade advanced (unresectable locally advanced or distant metastatic) neuroendocrine tumors were randomized comparing $10 \mathrm{mg}$ per day oral everolimus with placebo, both in conjunction with $30 \mathrm{mg}$ intramuscular octreotide LAR every 28 days. Primary endpoint was progression-free survival and disease progression established by radiological assessment within the past 12 months. Though 357 participants discontinued study treatment and one was lost to follow-up, median progression-free survival was $16 \cdot 4$ (95\% CI $13 \cdot 7-21 \cdot 2)$ months in the everolimus plus octreotide LAR group and $11.3(8.4-14.6)$ months in the placebo plus octreotide LAR group (hazard ratio $0 \cdot 77,95 \%$ CI $0 \cdot 59-1 \cdot 00$; one-sided log-rank test $\mathrm{p}=0 \cdot 026)$. The study did not meet its primary endpoint of PFS as assessed by independent radiological review ( $\mathrm{p}=0.026$ vs. $\mathrm{p}=0.0246$ predefined) (hazard ratio $=0.77$ [ $95 \%$ CI, 0.59 to 1.00$]$ ). However inconsistencies were found between analyses of radiology scans, which resulted in censoring of patients from the trial. These imbalances and the censoring of data seem to favor the control arm and may have impacted the outcome of the study. Additional analyses to adjust for imbalances in the treatment arms show everolimus plus octreotide LAR significantly reduced risk of disease progression $(\mathrm{HR}=0.60[95 \%$ CI, 0.44 to 0.84$]$ ).

Similarly, in a Phase III prospective, double-blind, randomized, parallel group, placebo-controlled, multicenter study (RADIANT-3), [54] efficacy and safety of everolimus plus BSC versus placebo plus BSC were compared in 410 patients with advanced, low- or intermediate-grade pancreatic NET. Patients were randomized 1:1 to receive either everolimus $10 \mathrm{mg}$ once-daily $(\mathrm{n}=207)$ or daily placebo $(n=203)$ orally, both in conjunction with BSC. The primary endpoint was PFS. Secondary endpoints include safety, objective response rate (confirmed according to RECIST), du- ration of response and overall survival. The median progression-free survival was 11.0 months with everolimus as compared with 4.6 months with placebo (hazard ratio for disease progression or death from any cause with everolimus, 0.35; $95 \%$ confidence interval [CI], 0.27 to 0.45 ; $\mathrm{P}<0.001$ ), representing a $65 \%$ reduction in the estimated risk of progression or death. Estimates of the proportion of patients who were alive and progression-free at 18 months were 34\% (95\% CI, 26 to 43) with everolimus as compared with 9\% (95\% CI, 4 to 16) with placebo.

\section{IMMUNOTHERAPY}

Immunotherapy either alone or in combination with other agents, is generally recommended as a second-line approach in patients with functioning GI-NETs and low proliferative index [55]. Interferon- $\alpha$ is the most widely studied and has been reported to have an antiproliferative effect on tumor cells, achieving a long-lasting biological response and effective symptomatic relief in a significant proportion of patients. However, in cases of neuroendocrine tumors, median biological and tumor responses of only $44 \%$ and $11 \%$ have been reported from pooled data from 13 series (total 383 patients). In addition, cessation of therapy due to severe side effects e.g. fevers, fatigue, anorexia and weight loss is common. However, there is conflicting evidence as to its efficacy, and there is some evidence it may have greater effect in tumors with low mitotic rate. It is difficult to recommend use of alpha-interferon as a primary therapy for unresectable NET with liver metastases, but perhaps it may yet have a limited role if used in combination with other therapies [56].

\section{ANGIOGENESIS INHIBITORS}

Sunitinib, sorafinib and bevacizumab have been applied as single agent in NETs with varying response rates of 5\%$15 \%$. Raymond et al [57] compared sunitinib with placebo in patients with pancreatic endocrine tumors and demonstrated a significantly larger PFS, (11.4 months versus 5.5 months, $P=0.0001)$.

\section{MEDICAL MANAGEMENT}

Medical management plays the most important role in the management of NETs with liver metastasis. As curative surgery is possible in minority of the patients, most require long term medical management to relieve symptoms and, and to suppress tumor growth and spread. Even when curative surgery is possible, patients need medical management during pre-operative and intra-operative period to prevent carcinoid crisis.

Somatostatin is an endogenous inhibitor of various hormones secreted from the endocrine system, but has limited clinical use due to its short half-life ( $<3 \mathrm{~min})$. Therefore, specific somatostatin analogues have been developed that act as receptor agonists and block hormone release. Octreotide, the first somatostatin analogue available commercially, is an $\mathrm{sst}_{2}$-preferring agonist and also has moderate affinity for $\mathrm{sst}_{3}$ and sst $_{5}$. It has a different chemical structure from somatostatin and a much longer half-life of 2 hours. Controlled trial with long acting octreotide (octreotide LAR) showed a significant decrease in the number of daily stools 
(42\%) and an $84 \%$ reduction in the incidence of flushing. In addition, complete or partial symptom control was achieved in $66 \%$ of patients receiving octreotide LAR 10-30 $\mathrm{mg} / \mathrm{month}$. Octreotide LAR also decreased 5-HIAA levels by $50 \%$ [58]. The mechanism by which somatostatin analogues normalize bowel function is not yet clear; however, it is thought to involve inhibition of gut hormone secretion. Studies have highlighted the antiproliferative effects of octreotide in patients with NETs and shown that approximately twothirds experience stable disease for up to 5 years, although objective tumor responses are uncommon (5\% of patients) [59]. The double-blind, placebo-controlled randomized study (PROMID Trial) of octreotide LAR or placebo in patients with well-differentiated metastatic midgut NETs demonstrated that octreotide LAR $30 \mathrm{mg} / \mathrm{month}$ more than doubled the time to tumor progression compared with placebo ( $P=$ 0.000072), in both functioning and non-functioning NETs. The PROMID study demonstrated that the benefits of octreotide treatment in NETs are independent of functionality, CgA level, Karnofsky performance status or age [60]. Based on the PROMID data, The National Comprehensive Cancer Network has recommended to include octreotide LAR as a management option for asymptomatic patients with recurrent, unresectable metastatic NETs.

Lanreotide was the second analogue available and has a similar binding profile to octreotide. The effects of lanreotide SR (administered every 7 - 14 days) on symptom relief are comparable to those of octreotide. A newer formulation of lanreotide (lanreotide autogel) that allows for monthly administration is also available. One study has shown that patients who received lanreotide autogel for 6 months, $65 \%$ achieved $\mathrm{a} \geq 50 \%$ reduction in flushing episodes, and $18 \%$ had $\mathrm{a} \geq 50 \%$ reduction in diarrhea episodes. The biochemical response rate observed with lanreotide is also comparable to that of octreotide, with response being greater in patient's naïve to somatostatin analogue therapy [61]. Patients may be stabilized with octreotide (short acting) for 10-28 days before converting them to long acting somatostatin analogues. Escalation of dose is often needed over time.

Patients with NETs receiving octreotide and lanreotide may experience a loss of response (the 'escape from response' phenomenon or tachyphylaxis) 6-18 months after the initiation of treatment. It has led to interest to develop multi-receptor ligand somatostatin analogues that could be as effective and well tolerated in patients who experience an escape from response. Pasireotide, a novel multi-receptor ligand analogue, may fulfill this role due to its 40-fold higher affinity and 158-fold higher functional activity for sst ${ }_{1-3}$ and sst $_{5}$ receptors $[62,63]$. Preliminary data are promising, with effective control of diarrhea and flushing observed in NET patients refractory or resistant to octreotide LAR [64]. A phase III study of pasireotide LAR versus octreotide LAR is ongoing in patients with metastatic carcinoid tumors (GINETs) whose disease-related symptoms are inadequately controlled by somatostatin analogues. Recently, interferon- $\alpha$ and octreotide have been advocated for the treatment of metastatic carcinoid.

\section{RADIATION THERAPY}

This is a useful palliative option for symptomatic patients with inoperable or metastatic tumor. Carcinoid tumors are not very radiosensitive, but external beam radiotherapy may be helpful in patients with painful bone metastases [65], and there has been a suggestion that some secondary deposits in the liver and elsewhere shrink in response to radiotherapy [66].

However, systemic receptor-targeted therapy (peptide receptor radiotherapy-PRRT) has provided suggest objective response rates of $30 \%-40 \%$ with disease stabilization in $40 \%$ of patients with unresectable somatostatin receptorpositive NETs. [67,68]. No randomized controlled trials have been performed.

\section{NEWER APPROACHES}

Treatment of metastatic disease has improved significantly with the addition of several new agents, including tyrosine kinase inhibitors, mammalian target of rapamycin inhibitors, and yttrium-90-DOTA (1,4,7,10-tetraazacyclo-dodecane1,4,7,10-tetraacetic acid) and lutetium-177-DOTA octreotate. Newer data are emerging about the potential role of the cell signaling transduction agents, which affect tyrosine kinase and other molecular markers, and important clinical trials are about to start. Imatinib has been used for carcinoid tumors but no adequate data are available. Trials using vaccines against various peptides are planned. Despite significant advances in the understanding and management of GEPNETs, the survival of patients remains largely unchanged and there remains a need for the development of national and international research collaborations to spearhead future efforts.

\section{CONCLUSION}

Incidence of Neuroendocrine tumors is gradually increasing and despite increased consciousness and diagnostic facilities, most patients present with metastasis, liver being the second commonest site. In the presence of liver metastasis, management guidelines of NETs may be considered as:

- The choice of treatment depends on the symptoms, stage of disease, degree of uptake of radionuclide, and histological features of the tumor.

- Curative surgery should be considered in those with liver metastases and potentially resectable disease.

- Where abdominal surgery is undertaken and long term treatment with SMS analogues is likely, cholecystectomy should be considered.

- Debulking surgery should be considered in those patients in whom curative surgery is not possible.

- For patients who are not fit for surgery, the aim of treatment is to improve and maintain an optimal quality of life.

- Treatment choices for non-resectable disease include SMS analogues, biotherapy, radionuclides, ablation therapies, and chemotherapy.

- External beam radiotherapy may relieve bone pain from metastases.

- Chemotherapy may be used for inoperable or metastatic pancreatic and bronchial tumors, or poorly differentiated NETs. 
In the future more sensitive biomarkers will be developed and molecular imaging will become cornerstones in the management of NETs. Personalized treatment based on tumor biology and molecular genetics will be available and will also include completely new therapeutics, based on the unique features of NETs.

\section{ACKNOWLEDGEMENT}

None declared.

\section{CONFLICIT OF INTEREST}

None declared.

\section{REFERENCES}

[1] Pictet RL, Rall LB, Phelps P, Rutter WJ. The neural crest and the origin of the insulin-producing and other gastrointestinal hormoneproducing cells. Science 1976; 191: 191-2.

[2] Lubarsch O. Über den primären Krebs des Ileum, nebst Bemerkungen über das gleichzeitige Vorkommen von Krebs und Tuberkolose. Virchows Arch 1888; 111: 280-317.

[3] Yao JC, Hassan M, Phan A, et al. One hundred years after 'carcinoid': epidemiology of and prognostic factors for neuroendocrine tumors in 35825 cases in the United States. J Clin Oncol 2008; 26: 3063-72.

[4] Modlin IM, Oberg K, Chung DC, et al. Gastroenteropancreatic neuroendocrine tumors. Lancet Oncol 2008; 9: 61-72.

[5] Kulke MH, Mayer RJ. Carcinoid tumors. N Engl J Med 1999; 340: 858-68.

[6] Modlin IM, Lye KD, Kidd M. A 5-decade analysis of 13,715 carcinoid tumors. Cancer 2003; 97: 934-59.

[7] Metz DC, Jensen RT. Endocrine tumors of the gastrointestinal tract and pancreas. In: Rustgi AK, Ed. Gastrointestinal cancers. Edinburgh: WB Saunders 2003; pp. 618-20.

[8] Van Gompel JJ, Sippel RS, Warner TF, Chen H. Gastrointestinal carcinoid tumors: factors that predict outcome. World J Surg 2004; 28: 387-92.

[9] Ohrvall U, Eriksson B, Juhlin C, et al. Method for dissection of mesenteric metastases in mid-gut carcinoid tumors. World J Surg 2000; 24: 1402-8.

[10] Pelage J-P, Soyer P, Boudiaf M, et al. Carcinoid tumors of the abdomen: CT features. Abdom Imaging 1999; 24: 240-5.

[11] Bader TR, Semelka RC, Chiu VC, et al. MRI of carcinoid tumors: spectrum of appearances in the gastrointestinal tract and liver. $\mathrm{J}$ Magn Reson Imaging 2001; 14: 261-9.

[12] Corleto VD, Panzuto F, Falconi M, et al. Digestive neuroendocrine tumors: diagnosis and treatment in Italy: a survey by the Oncology Study Section of the Italian Society of Gastroenterology (SIGE). Dig Liver Dis 2001; 33: 217-21.

[13] Zimmer T, Scherubl H, Faiss S, et al. Endoscopic ultrasonography of neuroendocrine tumors. Digestion 2000; 62(Suppl 1): 45-50.

[14] Frucht H, Doppman JL, Norton JA, et al. Gastrinomas: comparison of MR imaging with CT, angiography, and US. Radiology 1989; 171: 713-7.

[15] Rubin J, Ajani J, Schirmer W, et al. Octreotide acetate long-acting formulation versus open-label subcutaneous octreotide acetate in malignant carcinoid syndrome. J Clin Oncol 1999; 17: 600-6.

[16] Montravers F, Grahek D, Kerrou K, et al. Can fluorodihydroxyphenylalanine PET replace somatostatin receptor scintigraphy in patients with digestive endocrine tumors? J Nucl Med 2006; 47: 1455-62.

[17] Orlefors H, Sundin A, Garske U, et al. Whole-body (11)C-5hydroxytryptophan positron emission tomography as a universal imaging technique for neuroendocrine tumors: comparison with somatostatin receptor scintigraphy and computed tomography. J Clin Endocrinol Metab 2005; 90: 3392-400.

[18] Modlin IM, Oberg K, Chung DC, et al. Gastroenteropancreatic neuroendocrine tumors. Lancet Oncol 2008; 9: 61-72.

[19] Gibril F, Reynolds JC, Doppman JL, et al. Somatostatin receptor scintigraphy: its sensitivity compared with that of other imagingn methods in detecting primary and metastatic gastrinomas: a prospective study. Ann Intern Med 1996; 125: 26-34.

[20] Nicholson SA, Ryan MR. A review of cytologic findings in neuroendocrine carcinomas including carcinoid tumors with histologic correlation. Cancer 2000; 90: 148-61.

[21] Pirker RA, Pont J, Pohnl R, et al. Usefulness of chromogranin A as a marker for detection of relapses of carcinoid tumors. Clin Chem Lab Med 1998; 36: 837-40.

[22] Korse CM, Bonfrer JM, Aaronson NK, et al. Chromogranin A as an alternative to 5-hydroxyindoleacetic acid in the evaluation of symptoms during treatment of patients with neuroendocrine tumors. Neuroendocrinology 2009; 89: 296-301.

[23] Yao JC, Lombard-Bohas C, Baudin E, et al. Daily oral everolimus activity in patients with metastatic pancreatic neuroendocrine tumors after failure of cytotoxic chemotherapy: a phase II trial. J Clin Oncol 2010; 28: 69-76.

[24] Sarmiento JM, Que FG. Hepatic surgery for metastases from neuroendocrine tumors. Surg Oncol Clin N Am 2003; 12: 231-42.

[25] Hodul PJ, Strosberg JR, Kvols LK. Aggressive surgical resection in the management of pancreatic neuroendocrine tumors: when is it indicated? Cancer Control 2008; 15: 314-21.

[26] Yao KA, Talamonti MS, Nemcek AA, et al. Indications and results of liver resection and hepatic chemoembolization for metastatic gastrointestinal neuroendocrine tumors. Surgery 2001; 130: 677-85.

[27] Que FG, Nagorney DM, Batts KP, et al. Hepatic resection for metastatic neuroendocrine carcinomas. Am J Surg 1995; 169: 3642.

[28] Grazi GL, Cescon M, Pierangeli F, et al. Highly aggressive policy of hepatic resections for neuroendocrine liver metastases. Hepatogastroenterol 2000; 47: 481-6.

[29] Iwao M, Nakamuta M, Enjoji M, et al. Primary hepatic carcinoid tumor: case report and review of 53 cases. Med Sci Monit 2001; 7: 746-50.

[30] Sarmiento JM, Heywood G, Rubin J, et al. Surgical treatment of neuroendocrine metastases to the liver: a plea for resection to increase survival. J Am Coll Surg 2003; 197: 29-37.

[31] Routley D, Ramage JK, McPeake J, et al. Orthotopic liver transplantation in the treatment of metastatic neuroendocrine tumors of the liver. Liver Transpl Surg 1995; 1: 118-21.

[32] Le Treut YP, Delpero JR, Dousset B, et al. Results of liver transplantation in the treatment of metastatic neuroendocrine tumors: a 31-case French multicentric report. Ann Surg 1997; 225: 355-64.

[33] Schweizer RT, Alsina AE, Rosson R, Bartus SA. Liver transplantation for metastatic neuroendocrine tumors. Transplant Proc 1993; 25: 1973.

[34] Drougas JG, Anthony LB, Blair TK, et al. Hepatic artery chemoembolization for management of patients with advanced metastatic carcinoid tumors. Am J Surg1998; 175: 408-12.

[35] Strosberg JR, Nasir A, Hodul PJ, Kvols LK. Biology and treatment of metastatic gastrointestinal neuroendocrine tumors. Gastrointest Cancer Res 2008; 2: 113-25.

[36] Kennedy AS, Dezarn WA, McNeillie P, et al. Radioembolization for unresectable neuroendocrine hepatic metastases using resin 90Y-microspheres: early results in 148 patients. Am J Clin Oncol 2008; 31: 271-9.

[37] Wessels FJ, Schell SR. Radiofrequency ablation treatment of refractory carcinoid hepatic metastases. J Surg Res 2001; 95: 8-12.

[38] Gillams AR. Radiofrequency ablation in the management of liver tumors. Eur J Surg Oncol 2003; 29: 9-16.

[39] Siperstein AE, Berber E. Cryoablation, percutaneous alcohol injection, and radiofrequency ablation for treatment of neuroendocrine liver metastases. World J Surg 2001; 25: 693-6.

[40] Siperstein A, Garland A, Engle K, et al. Laparoscopic radiofrequency ablation of primary and metastatic liver tumors. Technical considerations. Surg Endosc 2000; 14: 400-5.

[41] Eriksson BK, Larsson EG, Skogseid BM, et al. Liver embolizations of patients with malignant neuroendocrine gastrointestinal tumors. Cancer 1998; 83: 2293-301.

[42] Castellani MR, Chiti A, Seregni E, Bombardieri E. Role of 131Imetaiodobenzylguanidine (MIBG) in the treatment of neuroendocrine tumors. Experience of the National Cancer Institute of Milan. Q J Nucl Med 2000; 44: 77-87.

[43] Moertel CG, Lefkopoulo M, Lipsitz S, et al. Streptozocindoxorubicin, streptozocin-fluorouracil, or chlorozotocin in the treatment of advanced islet-cell carcinoma. N Engl J Med 1992; 326: 519-23. 
[44] Moertel CG, Kvols LK, O’Connell MJ, et al. Treatment of neuroendocrine carcinomas with combined etoposide and cisplatin: evidence of major therapeutic activity in the anaplastic variants of these neoplasms. Cancer 1991; 68: 227-32.

[45] Pelley RJ, Bukowski RM. Recent advances in systemic therapy for gastrointestinal neuroendocrine tumors. Curr Opin Oncol 1999; 11: 32-7.

[46] Ritzel U, Leonhardt UF, Stockmann F, et al. Treatment of metastasized midgut carcinoids with dacarbazine. Am J Gastroenterol 1995; 90: 627-31.

[47] Mitry E, Baudin E, Ducreux M, et al. Treatment of poorly differentiated neuroendocrine tumors with etoposide and cisplatin. $\mathrm{Br} \mathrm{J}$ Cancer 1999; 81: 1351-5.

[48] Mavligit GM, Pollock RE, Evans HL, et al. Durable hepatic tumor regression after arterial chemoembolization-infusion in patients with islet cell carcinoma of the pancreas metastatic to the liver. Cancer 1993; 72: 375-80.

[49] Ekeblad S, Sundin A, Janson ET, et al. Temozolomide as monotherapy is effective in treatment of advanced malignant neuroendocrine tumors. Clin Cancer Res 2007; 13: 2986-91.

[50] Decristoforo C, Mather SJ, Cholewinski W, et al. 99mTcEDDA/HYNIC-TOC: a new 99mTc-labelled radiopharmaceutical for imaging somatostatin receptor-positive tumors; first clinical results and intra-patient comparison with 111In-labelled octreotide derivatives. Eur J Nucl Med 2000; 27: 1318-25.

[51] Kwekkeboom DJ, Bakker WH, Kam BL, et al. Treatment of patients with gastro-entero-pancreatic (GEP) tumors with the novel radiolabelled somatostatin analogue [(177)Lu-DOTA(0),Tyr(3)] octreotate. Eur J Nucl Med Mol Imaging 2003; 30: 417-22.

[52] Kwekkeboom DJ, de Herder WW, Kam BL, et al. Treatment with the radiolabeled somatostatin analog $\left[{ }^{177} \mathrm{Lu}\right.$-DOTA, $\left.\mathrm{Tyr}^{3}\right]$ octreotate: toxicity, efficacy, and survival. J Clin Oncol 2008; 26 (13): 212430.

[53] Pavel ME, Hainsworth JD, Baudin E, et al. Everolimus plus octreotide long-acting repeatable for the treatment of advanced neuroendocrine tumors associated with carcinoid syndrome (RADIANT-2): a randomised, placebo-controlled, phase 3 study. Lancet 2011; 378(9808): $2005-12$.

[54] Yao JC, Shah MH, Ito T, et al. Everolimus for advanced pancreatic neuroendocrine tumors everolimus for advanced pancreatic neuroendocrine tumors. N Engl J Med 2011; 364: 514-23.

[55] Eriksson B, Kloppel G, Krenning E, et al. Consensus guidelines for the management of patients with digestive neuroendocrine tumorswell-differentiated jejunal-ileal tumor/carcinoma. Neuroendocrinology 2008; 87: 8-19.
[56] Oberg K. Interferon in the management of neuroendocrine GEPtumors: a review. Digestion 2000; 62(Suppl. 1): 92-7.

[57] Raymond E, Niccoli P, Bang YJ, et al. Updated results of the phase III trial of sunitinib v.s. placebo for treatment of advanced pancreatic neuroendocrine tumors. ASCO Gastrointestinal Cancer Symposium 2010 Jan 22-24 Orland, FL: USA, 2010.

[58] Schmid HA, Schoeffter P. Functional activity of the multiligand analog SOM230 at human recombinant somatostatin receptor subtypes supports its usefulness in neuroendocrine tumors. Neuroendocrinology 2004; 80(Suppl. 1): 47-50.

[59] Kulke MH. Neuroendocrine tumors: is there a standard treatment? Gastrointest Cancer Res 2008; 2: 152-3.

[60] Yao JC, Phan AT, Chang DZ, et al. Efficacy of RAD001 (everolimus) and octreotide LAR in advanced low- to intermediategrade neuroendocrine tumors: results of a phase II study. J Clin Oncol 2008; 26: 4311-8.

[61] Ruszniewski P, Ish-Shalom S, Wymenga M, et al. Rapid and sustained relief from the symptoms of carcinoma syndrome; results from an open 6-month study of the 28-day prolonged-release formulation of lanreotide. Neuroendocrinology 2004; 80: 244-51.

[62] Anthony LB, Martin W, Delbeke D, Sandler M. Somatostatin receptor imaging: predictive and prognostic considerations. Digestion 1996; 57(Suppl. 1): 50-3.

[63] Grozinsky-Glasberg S, Shimon I, Korbonits M, Grossman AB. Somatostatin analogues in the control of neuroendocrine tumors: efficacy and mechanisms. Endocr Relat Cancer 2008; 15: 701-20.

[64] Kvols L. Safety and efficacy of pasireotide (SOM230) in patients with metastatic carcinoid tumors refractory or resistant to octreotide LAR: results of a phase II study. ASCO GI Cancer Symposium Abstract No 1712006.

[65] Imtiaz KE, Monteith P, Khaleel A. Complete histologic regression of metastatic carcinoid tumor after treatment with octreotide. Clin Endocrinol 2000; 53: 755-8.

[66] Chakravarthy A, Abrams RA. Radiation therapy in the management of patients with malignant carcinoid tumors. Cancer 1995; 75: 1386-90.

[67] Forrer F, Valkema R, Kwekkeboom DJ, de Jong M, Krenning EP. Neuroendocrine tumors: peptide receptor radionuclide therapy. Best Pract Res Clin Endocrinol Metab 2007; 21: 111-29.

[68] Valkema R, Pauwels S, Kvols LK, et al. Survival and response after peptide receptor radionuclide therapy with [90Y-DOTA0, Tyr3] octreotide in patients with advanced gastroenteropancreatic neuroendocrine tumors. Semin Nucl Med 2006; 36: 147-56.

(C) Kanti et al.; Licensee Bentham Open.

This is an open access article licensed under the terms of the Creative Commons Attribution Non-Commercial License (http://creativecommons.org/licenses/ by-nc/3.0/) which permits unrestricted, non-commercial use, distribution and reproduction in any medium, provided the work is properly cited. 